\title{
Micropillar compression inside zirconia degraded layer
}

\section{Abstract}

In the dental field, Yttria-doped Tetragonal Polycrystalline Zirconia (Y-TZP) plays an important role due to its high mechanical properties, good aesthetics and bio-inert behavior. Surface mechanical properties are crucial for Y-TZP especially when it comes to osseointegration or contact loading, since it may undergo a spontaneous aging phenomenon when exposed to humid environment, creating a thin degraded layer that affects surface integrity. In this work, polished samples of Y-TZP have been artificially aged, and micropillars with diameters in the range $3.3 \mu \mathrm{m}-0.3 \mu \mathrm{m}$ have been milled by FIB inside the degraded layer and in the reference non-aged surface. Reproducible stress-strain curves were obtained by testing the micropillars in compression, demonstrating that this technique is suitable for assessing the degraded surface properties. Mechanical and failure behavior are significantly different for the degraded micropillars due to the presence of microcracks, while important differences are observed when reducing the micropillar diameter.

Keywords: Zirconia; micropillar compression; Low Temperature Degradation; small-scale testing.

\section{Introduction}

Structural zirconia ceramics are widely and increasingly used in dentistry due to their outstanding properties, especially in terms of fracture toughness $\left(K_{I c}\right)$, biocompatibility and aesthetics [1]. The main mechanism responsible for the relative high value of $K_{I c}$ is transformation toughening, that is, the transformation of the metastable tetragonal $(t)$ phase into monoclinic phase $(m)$ in front of the crack tip triggered by the high local stress generated under loading. During this transformation, a local volume expansion of approximately $4.5 \%$ takes place, inducing a compressive component at the crack tip that hinders its propagation. The result is a 
net increase in $K_{I c}$, which depends on different variables related to the particular alloying system and microstructure of zirconia [2].

1 Here the attention is addressed to yttria-stabilized tetragonal polycrystalline zirconia (3Y-TZP), a system

2

with very fine microstructure composed almost entirely of tetragonal grains with size of approximately 350 $\mathrm{nm}$. The $3 \mathrm{~mol} \%$ yttria content is the result of optimization in order to attain high mechanical strength. Approximately $1200 \mathrm{MPa}$ flexure strength is measured for a standard processed material, which is the highest among single-phase ceramics, and a considerable $K_{I c}$ of $\sim 4.5 \mathrm{MPa} \vee \mathrm{m}$.

At present, 3Y-TZP is being extensively used in dentistry for the production of dental crowns, bridges, abutments, dentures and dental implants [3]. The microstructure, topography, and mechanical properties of the surface are of extreme importance for all these applications, especially when it comes to load transfer by contact loading. In this sense, the long-term surface stability of zirconia became an issue after the discovery that hydrothermal degradation, an aging phenomenon that affects the surface of zirconia when exposed to humid environment and temperatures around $250{ }^{\circ} \mathrm{C}$, may also take place at much lower temperatures including that of the human body. During aging, due to the diffusion of water species, the tetragonalmonoclinic $(t-m)$ transformation starts to occur spontaneously on the surface through a nucleation-andgrowth mechanism. After long exposure, grain boundary microcracks are formed within a thin superficial layer and monoclinic uplifts appear on the surface producing an increase in roughness [4]. The process is also accompanied by grain pull-out, a considerable decrease in surface hardness that can be measured by nanoindentation and the deterioration of scratch resistance [5-7]. The extent of transformation can be observed, and eventually measured, in terms of fraction of monoclinic phase content from its first appearance on some isolated grains [8] to the progression through the first superficial layers [9] and into the volume [10].

Even though all these aspects have been widely documented by accelerated aging tests in water vapor at the standard sterilization temperature and pressure, there is still lack of knowledge on how mechanical properties are modified inside the transformed layer, which is generally few $\mu \mathrm{m}$ in thickness after long exposure to the human body environment. It was observed by TEM that degradation microcracks develop anisotropically, with the crack planes mainly orientated along planes roughly parallel to the free surface [11]. The spatial distribution of microcracks in the degraded volume was also measured by means of focused ion beam (FIB) tomography [12], confirming the anisotropic distribution.

Small-scale testing refers to mechanical tests performed on a reduced volume of material, an approach that can shed some light on the mechanical behavior of the degraded surface of zirconia. A first and wellknown example of these techniques is represented by nanoindentation, which finds its limitation in the complex stress state surrounding the indenter tip. With the development of FIB and other high precision shaping techniques, coupled with the load-displacement resolution offered by the technology of nanoindenters, new methods started to appear. By milling small volume samples with suitable geometry, the response of materials to compression, bending, torsion and tension states could be probed. For more details 
about these techniques, see Legros et al. [13]. In metallic monocrystals, the main result of these studies is that the rule "the smaller being stronger" holds for many systems and models related to strain gradients and

interaction of dislocations with free surfaces have been developed for explaining this particular behavior $[14,15]$.

In the case of ceramic monocrystals, only few authors have used micropillar compression testing, often with the main objective of observing the existence of a brittle-ductile transition. In effect, a ductile behavior could be observed by reducing the sample size below some critical value that lies roughly between tens and hundreds of nanometers. Several materials conventionally known as "brittle" have shown plasticity features at the small scale: $\mathrm{Si}$ [16], $\mathrm{GaAs}$ [17], $\mathrm{MgO}$ [18] $\mathrm{Al}_{2} \mathrm{O}_{3}$ [19] and $\mathrm{SiC}$ [20]. At the same time, superelastic and shape-memory effects were recently observed by Lai et al. [21] during compression of tetragonal zirconia micropillars highly doped with Y and Ce. Size-dependent phenomena related to flow stress in ceramic micropillars have also been documented by Korte and Clegg [18], showing a similar behavior to metals once dislocations are activated by high stresses. Recently, the flexural response of polycrystalline microcantilevers milled by FIB inside the zirconia degraded layer has been studied by Camposilvan et al. [22], observing a significantly different behavior depending on the direction of the applied stress, thus confirming that the anisotropic damage affects the mechanical properties inside the layer.

In the present work, micropillar compression testing is applied to 3Y-TZP. Polycrystalline micropillars are milled by FIB from the surface of fully degraded and non-degraded zirconia, with the main objective of studying the effect of degradation on the mechanical behavior.

\section{Experimental}

Commercial spray-dried zirconia powder (TZ-3YSB-E, Tosoh Corp.) was pressed isostatically at $200 \mathrm{MPa}$ in a rod shape and sintered at $1450{ }^{\circ} \mathrm{C}$ in air inside a tubular furnace for 2 hours, obtaining a ceramic with density of $6.06 \pm 0.02 \mathrm{~g} / \mathrm{cm}^{3}(99.5 \pm 0.3 \%$ of the theoretical value) as measured by the Archimedes' method. The rod was cut into disks of. $\sim 1.5 \mathrm{~mm}$ thickness and these were ground and polished with diamond pastes down to less than $20 \mathrm{~nm}$ Ra. Few samples were exposed to artificial degradation in autoclave, under steam atmosphere at $134^{\circ} \mathrm{C}$ and 2 bar pressure, for 145 hours. The grain size was measured by the linear intercept method on polished and thermally etched $\left(1300^{\circ} \mathrm{C}\right)$ surfaces.

X-ray diffraction (XRD) patterns were collected before and after artificial degradation using a Bruker D8 Advance diffractometer with $\mathrm{Cu}$ k- $\alpha$ radiation and $\theta / 2 \theta$ configuration in the region $25^{\circ}-35^{\circ}$. The intensity of selected peaks was used to calculate the monoclinic volume fraction by applying the equation of Toraya et al. [23]. Hardness and indentation fracture (IF) toughness were measured by the Vickers indentation method with a load of $10 \mathrm{Kg}$. The IF toughness was calculated with the formula proposed by Niihara et al. [24]. Nano-hardness and elastic modulus were measured with a Berkovich indenter mounted on a MTS Nanoindenter XP equipped with a continuous stiffness measurement (CSM) module. 4 matrices of $3 \times 3$ nanoindentations were performed at random locations on the disks surface, with constant deformation speed 
of $0.05 \mathrm{~s}^{-1}$ to a penetration depth of $500 \mathrm{~nm}$, which was selected to get stable measurements within the superficial layer.

The degradation time was chosen in order to produce a superficial fully degraded layer with a thickness greater than $13 \mu \mathrm{m}$ [10]. In this way, micro-sized fully degraded samples could be obtained from the surface. For doing so, both degraded and reference disks were cut along the diameter and the cross-section was polished using diamond films on a tripod fixture (Struers A/S). The disk halves were mounted on inclinable holders for scanning electron microscopy (SEM) using high strength silver adhesive and coated with a few nanometers $\mathrm{Au} / \mathrm{Pd}$ layer to increase conductivity and avoid charging phenomena during milling.

The holders were introduced in a Zeiss Neon 40 dual beam microscope in order to mill by FIB micropillars with aspect ratio between 2:1 and 4:1 from the surface area adjacent to the cross-section edge. A major issue in this procedure is represented by the taper angle that forms when employing FIB to mill cross-sectional surfaces. A way to limit this effect is by milling the final shape with multiple steps, so that the last current can be small enough to allow careful focusing and stigmation. At the same time, the dwell time assigned to every pixel can be varied in order to optimize the milling time and the shape of the feature. The procedure was therefore optimized and micropillars were milled in two steps: in the first, a large $34 \mu \mathrm{m}$ diameter well was obtained with a considerable ion current. In the second step, lower currents were employed and the milling profile was tuned for each micropillar size. In this way, micropillars of $3.3 \mu \mathrm{m}$, $700 \mathrm{~nm}$ and $300 \mathrm{~nm}$ nominal diameter and reduced taper angle were obtained. Micropillars were always milled with their axis perpendicular to the surface of the disks.

After milling, a thin cyanoacrylate strip was deposited on the surface of samples close to the milling area and the SEM holder was mounted on the stage of a MTS XP nanoindenter equipped with a CSM module for testing. Compression tests were performed with a flat-punch diamond tip machined via FIB from a Berkovich indenter. The flat surface had an equilateral triangle shape with a side of $18 \mu \mathrm{m}$. In order to clean the tip and align the surface of the sample, several indentations were performed on the cyanoacrylate strip and the SEM holder was tilted until a clear, symmetrical shape could be seen with the built-in optical microscope of the nanoindenter. During a compression test, the micropillar well was first imaged with the microscope and, after selecting the target location, the sample stage moved under the indenter tip. A microscope-to-indenter calibration had to be performed before each test in order to load the desired micropillar with the center of the flat surface. The compression routine was designed so that thermal drift was measured in contact with the micropillar at the beginning of the test. Afterward, loading was carried out under load control, adjusting the loading rate to obtain a strain rate approximately constant, until failure of the micropillar. In order to appreciate the change in stiffness and detect irreversible components of the deformation, additional tests were performed by loading-unloading cycles between a minimum constant load and increasing peak loads until failure employing a CSM Ultra Nano Hardness Tester (CSM Instruments, Peseux, Switzerland). Micropillars were imaged by SEM after testing. 
During processing of load-displacement data, the displacement at every load value was recalculated by subtracting the correspondent thermal drift. The data were converted into stress-strain curves by taking into acco appearing in the upper part of the micropillar, where the diameter is slightly smaller and the applied stress is the maximum in the sample.

\section{Results}

Table 1 reports the grain size, HV10 hardness, nano-hardness, nano-elastic modulus, IF toughness (Niihara equation) as well as the amount of monoclinic phase in the reference as-sintered zirconia $(A S)$ and in the artificially degraded one $(D E G)$ measured by XRD. HV10 hardness and IF toughness were not measurable from $D E G$ disks since extensive chipping occurred during loading, as shown in Figure 1, which presents also the aspect of a cross-section of the degraded disk after polishing, revealing the thickness of the degraded layer. The DEG micropillars, with their axis perpendicular to the surface, were milled inside this layer. The actual dimensions of the micropillar tested and the associated nomenclature are reported in Table 2. An equal number of 6 pillars for each group are considered in the results.

Table 1. Initial properties of as-sintered and degraded zirconia

\begin{tabular}{lllllll}
\hline Material & $\begin{array}{l}\text { Grain Size } \\
(\mu \mathrm{m})\end{array}$ & $\begin{array}{l}\text { HV10 } \\
(\mathrm{GPa})\end{array}$ & $\begin{array}{l}\text { IF Toughness } \\
(\mathrm{MPa} \sqrt{\mathrm{m}})\end{array}$ & $\begin{array}{l}\text { Nano-Hardness } \\
(\mathrm{GPa})\end{array}$ & $\begin{array}{l}\text { Nano-E } \\
(\mathrm{GPa})\end{array}$ & $\begin{array}{l}\text { Monoclinic Cont. } \\
(\mathrm{Vol} \%)\end{array}$ \\
\hline$A S$-control & $0.32 \pm 0.03$ & $13.0 \pm 0.1$ & $4.9 \pm 0.1$ & $17.1 \pm 0.2$ & $248 \pm 7$ & 0 \\
Degraded & $0.32 \pm 0.03$ & -- & -- & $12.2 \pm 0.3$ & $189 \pm 11$ & $84 \pm 3$ \\
\hline
\end{tabular}

Table 2. Size and geometry of tested micropillars

\begin{tabular}{ccccc}
\hline $\begin{array}{c}\text { Sample } \\
\text { Name }\end{array}$ & Diameter $(\mu \mathrm{m})$ & Height $(\mu \mathrm{m})$ & Aspect Ratio $(\mathrm{d} / \mathrm{h})$ & Taper angle $\left({ }^{\circ}\right)$ \\
\hline$A S 0.3$ & $0.32 \pm 0.01$ & $1.25 \pm 0.17$ & $3.91 \pm 0.14$ & $2.4 \pm 0.2$ \\
$A S 0.7$ & $0.68 \pm 0.03$ & $2.06 \pm 0.11$ & $3.03 \pm 0.07$ & $1.7 \pm 0.2$ \\
$A S 3.3$ & $3.3 \pm 0.15$ & $8.12 \pm 0.32$ & $2.46 \pm 0.06$ & $2.6 \pm 0.1$ \\
$D E G 0.3$ & $0.31 \pm 0.01$ & $1.31 \pm 0.05$ & $4.23 \pm 0.05$ & $2.4 \pm 0.1$ \\
$D E G 0.7$ & $0.70 \pm 0.02$ & $1.80 \pm 0.10$ & $2.57 \pm 0.06$ & $1.5 \pm 0.1$ \\
$D E G 3.3$ & $3.0 \pm 0.22$ & $8.51 \pm 0.29$ & $2.84 \pm 0.08$ & $2.7 \pm 0.2$ \\
\hline
\end{tabular}

Figure 2 reports some of the stress-strain curves obtained from $D E G$ micropillars of different sizes, which can be compared with representative curves for the $A S$ material shown on the same plot. Only some of the curves for the $D E G$ material are represented for clarity. The scatter in the response was relatively small for DEG 3.3 and DEG 0.7 samples of the same size, where the failure stress and strain were markedly reduced with respect to the AS counterparts. A slight deviation from linearity in the monotonic tests could be appreciated as the stress got close to the failure value for these samples.

The situation is different for $D E G 0.3$ micropillars, where significant variability in terms of failure stress and strain was recorded among individual tests. Three curves are presented for DEG 0.3, showing that intermediate failure stresses were obtained, together with failure strains as high as for the AS micropillars in some cases. The stress-strain curves present strain bursts that contributed substantially to the total strain. 

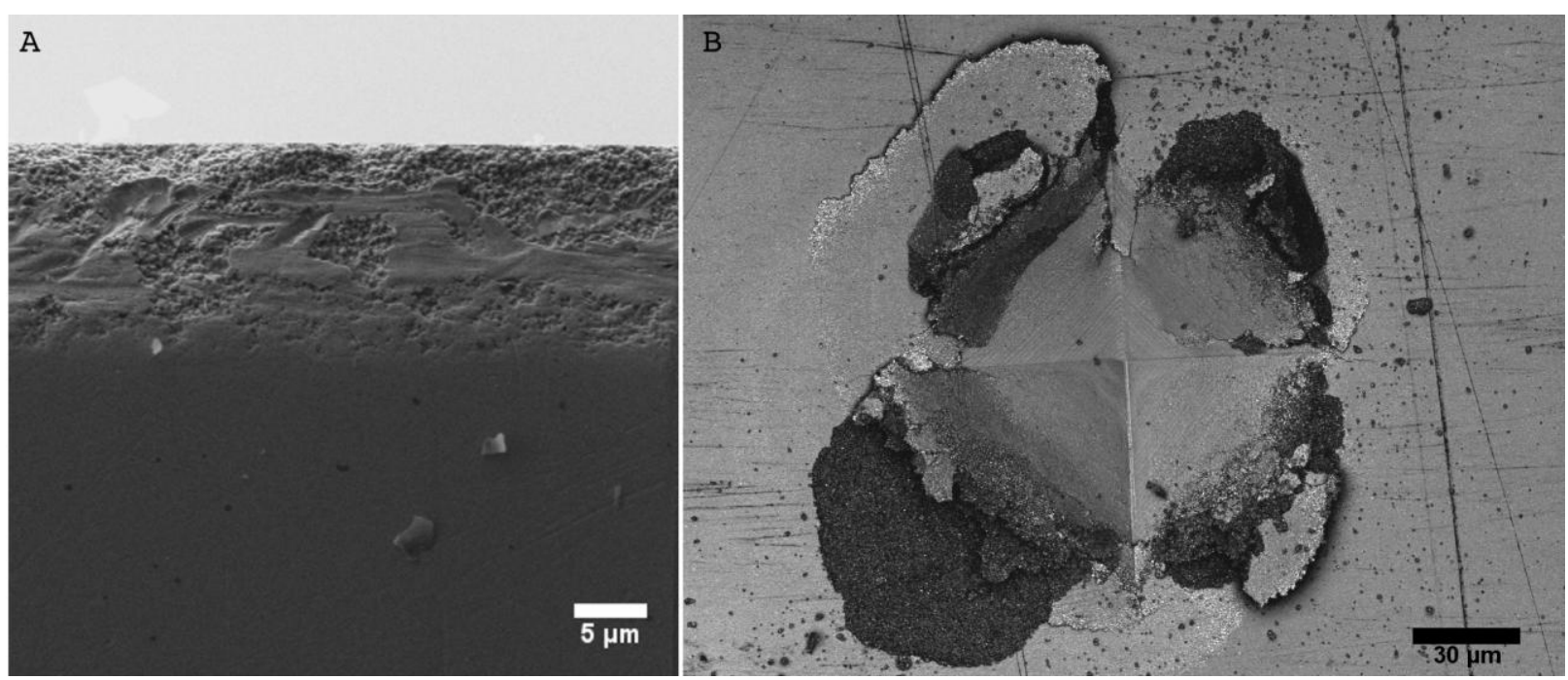

Figure 1. A: SEM image of the polished cross-section of the artificially degraded disk in the region where the micropillars were milled. The degraded layer presents a granular appearance. Traces of the action of grinding media are also visible inside the degraded layer. B: laser confocal scanning microscopy image of an HV10 indent on the surface of the degraded disk, showing the presence of chipping and debris and the absence of Palmqvist cracks.

On the same plot of Figure 2, the line corresponding to the theoretical elastic modulus of bulk polycrystalline non-degraded zirconia in the AS condition $(210 \mathrm{GPa})$ is shown, as well as the point of failure of the micropillars. It can be observed that the initial slopes of the compression curves of $D E G$ and $A S$ micropillars of $3.3 \mu \mathrm{m}$ and $0.7 \mu \mathrm{m}$ are significantly different by a factor close to 2 .

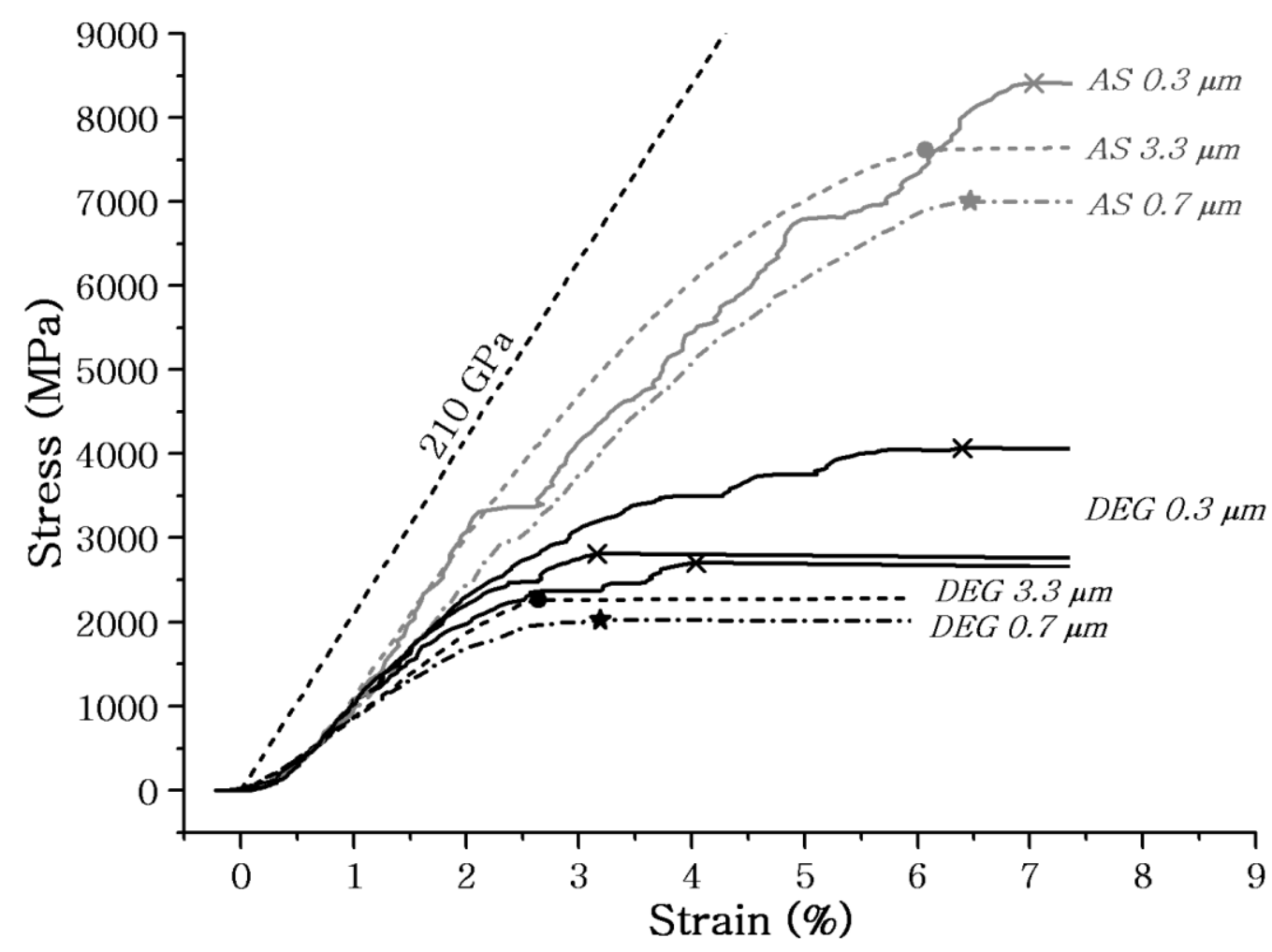

Figure 2. Stress-strain curves for degraded and non-degraded micropillars of various diameters. One representative curve is reported for all the samples but the DEG 0.3 ones, where 3 curves are presented to show the difference between individual tests. Symbols indicate micropillar failure. The ideal elastic modulus of tetragonal polycrystalline zirconia (210 GPa) is also plotted.

Figure 3 reports the stress-strain curves associated to loading-unloading tests at increasing peak stress for DEG 3.3 pillars. On unloading, a permanent deformation can be detected, which becomes more important as 
successive loading cycles, in a similar fashion as the unloading slope at the peak stress $\left(\mathrm{S}_{\mathrm{UP}}\right)$, where the increase is more marked. On the contrary, the unloading slope at low stresses $\left(\mathrm{S}_{\mathrm{U} 0}\right)$ decreases slightly with

increasing peak stress. It can also be observed that the corresponding unloading/loading hysteresis loops, indicated by the shaded area of the diagram in Fig. 4, are slightly open and that their loop width increases as the peak stress increases.
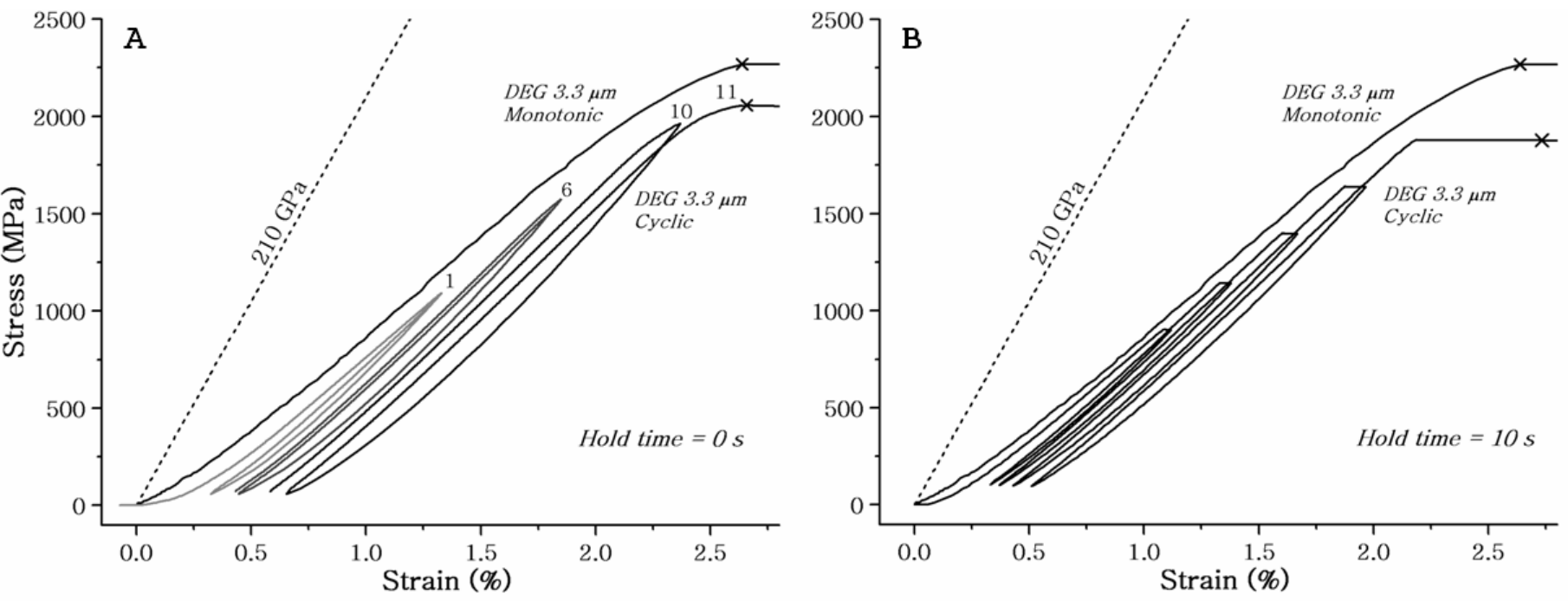

Figure 3. Stress-strain curves for DEG 3.3 micropillars by cyclic loading/unloading at increasing peak stress, with (B) and without (A) hold segment at the peak load. Crosses indicate micropillar failure. The ideal elastic modulus of tetragonal polycrystalline zirconia $(210 \mathrm{GPa})$ and a monotonic curve for a micropillar of similar diameter are also plotted.

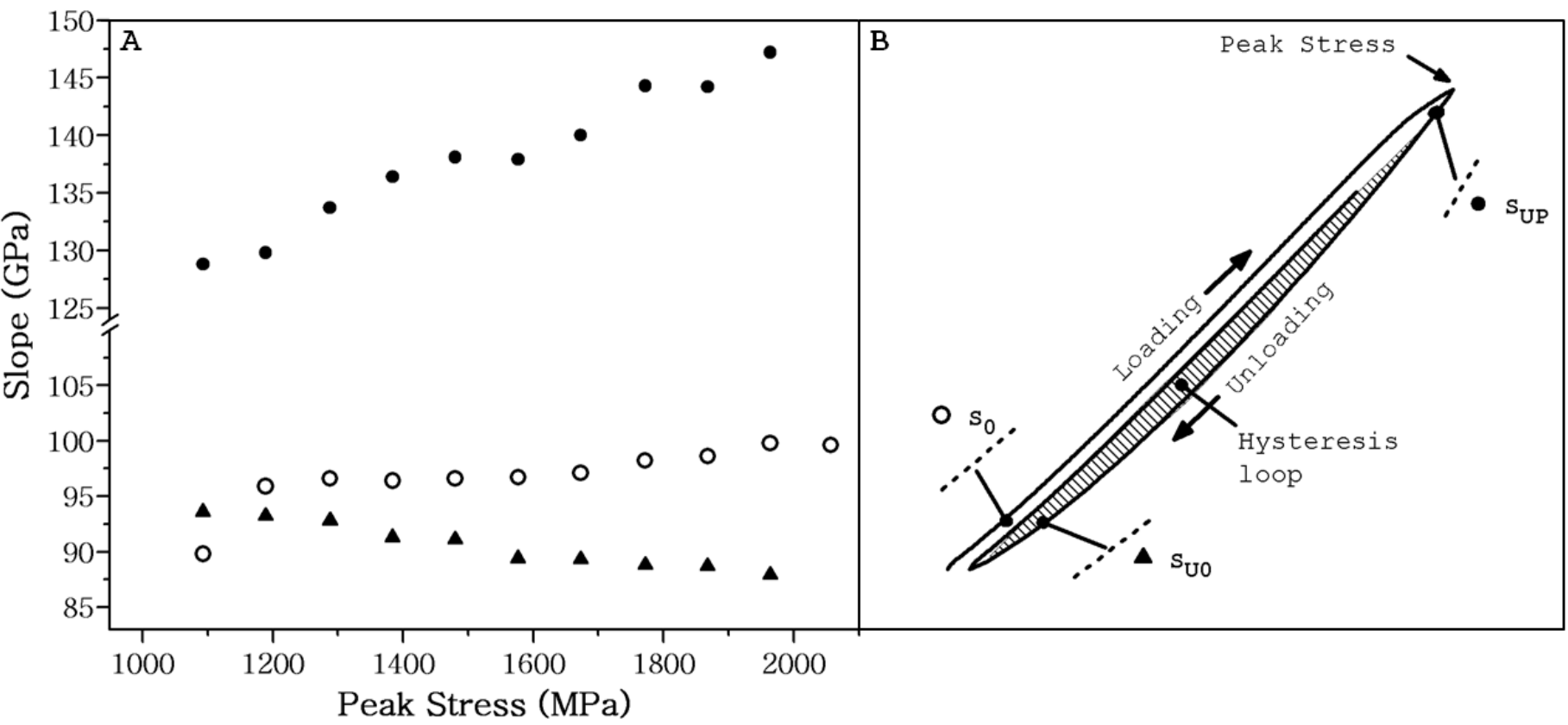

Figure 4. A: initial loading slope $\left(\mathrm{S}_{0}\right)$, unloading slope at peak load $\left(\mathrm{S}_{\mathrm{UP}}\right)$ and unloading slope at minimum load $\left(\mathrm{S}_{\mathrm{U} 0}\right)$ as a function of the peak load applied in each cycle during cyclic loading at increasing peak stress (Hold time $=0 \mathrm{~s}$ ). The diagram in $\mathrm{B}$ shows an example of loading cycle indicating where the slopes were measured.

The side view of two $0.7 \mu \mathrm{m}$ pillars before testing, milled respectively from $A S$ and $D E G$ surfaces, is presented in Fig. 5. In these images, the contrast has been enhanced to appreciate the shape and distribution of degradation cracks. The presence of thick dark lines reveals that some of the cracks are slightly open. 


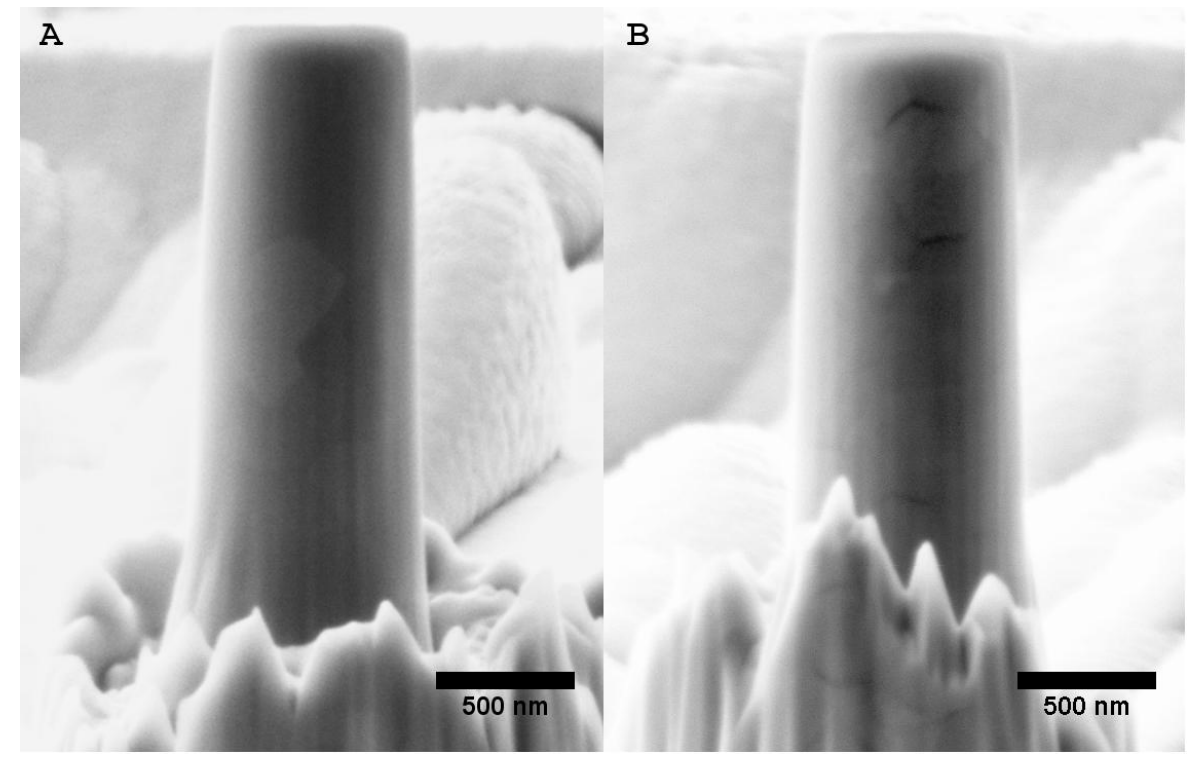

Figure 5. Lateral surface of one AS 0.7 (A) and one DEG 0.7 (B) micropillars before testing. Degradation-induced microcracks are clearly observed in the degraded pillar. The vertical striations are due to the milling procedure. Contrast has been enhanced in both pictures.

SEM images of degraded micropillars of two different sizes, before and after testing, are presented in Figures 6 and 7, where the fracture appearance can be appreciated.

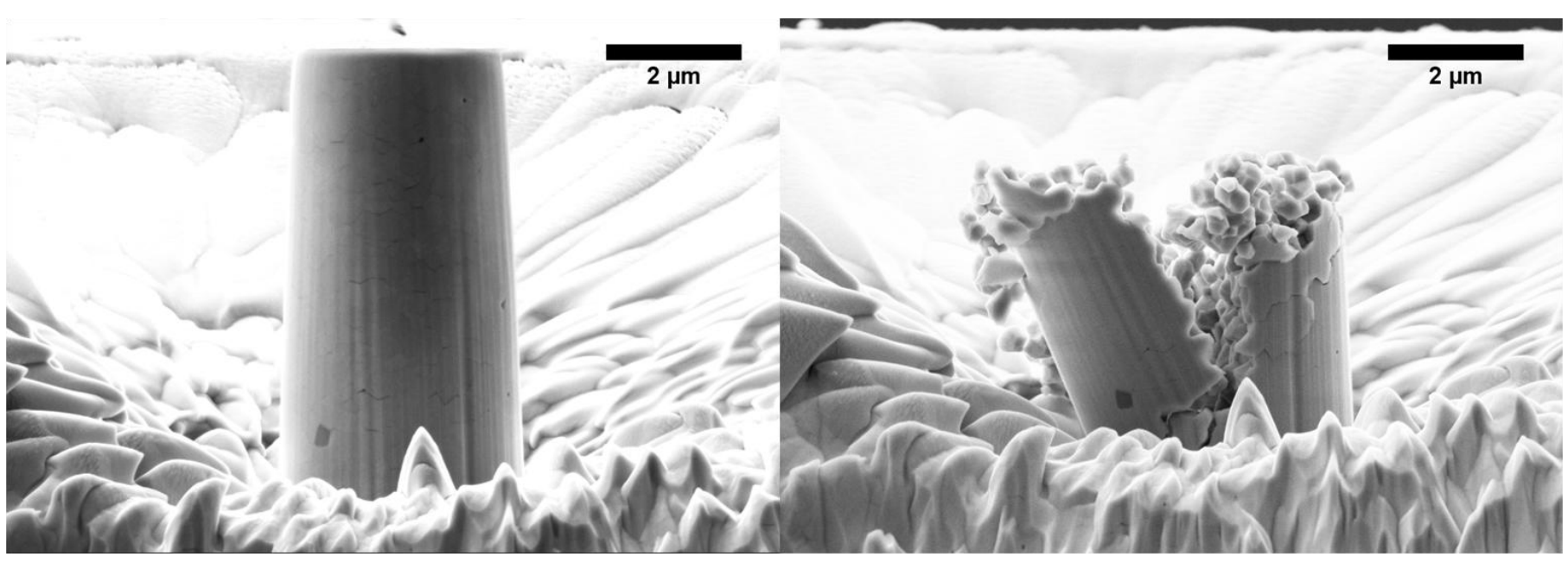

41 Figure 6. DEG $3.3 \mu \mathrm{m}$ micropillar before and after compression. The intergranular character of fracture can be appreciated on the 42 right picture.

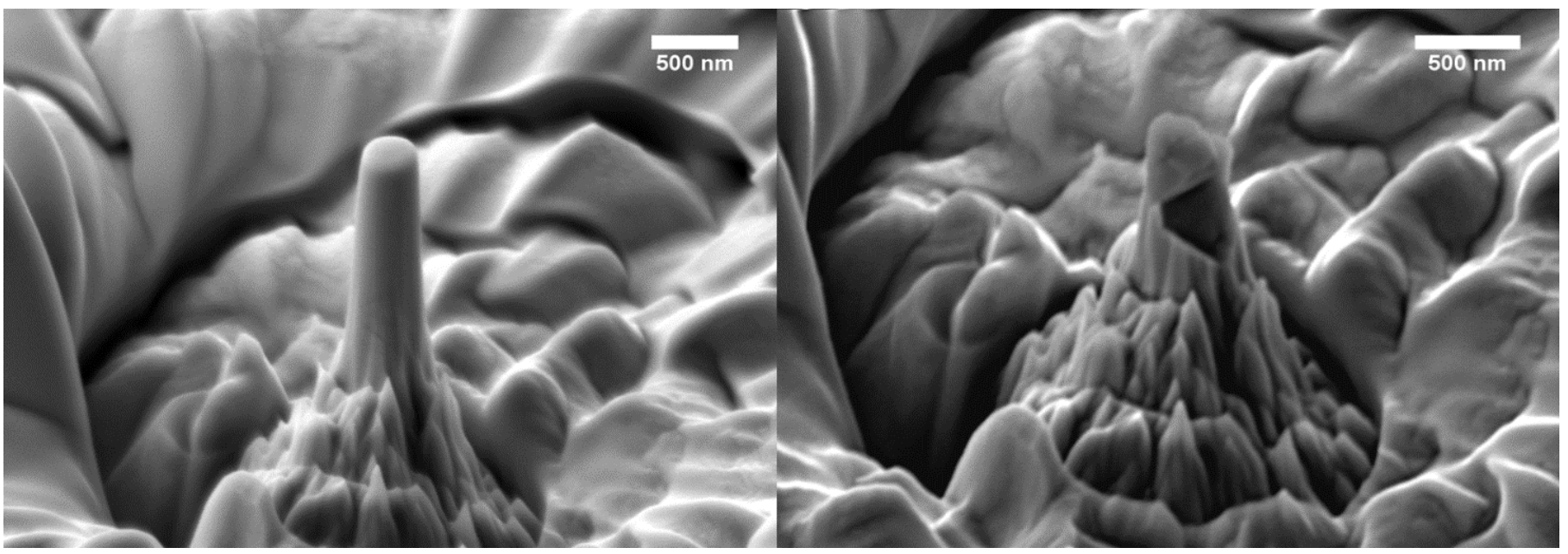

Figure 7. DEG 0.3 micropillar before and after compression. Grain pull-out during testing is evident in the picture on the right. 


\section{Discussion}

After artificial degradation, the fraction of monoclinic phase reached the saturation point of

1

approximately $85 \mathrm{vol} \%$ [25]. This limit is the result of the presence of about $10-15$ vol\% of nontransformable cubic phase developed during sintering. While HV10 could not give quantitative information about the mechanical alteration induced by the aging phenomenon in the $D E G$ surface due to extensive chipping and debris formation during loading, the results of nanoindentation depict better the change in hardness and elastic modulus in the superficial thickness. These values drop from $\sim 17 \mathrm{GPa}$ to $\sim 12 \mathrm{GPa}$ and from $\sim 248 \mathrm{GPa}$ to $\sim 189 \mathrm{GPa}$, respectively. In Ref. [6], such changes were ascribed to the presence of the microcracks network, which reduces the contact stiffness. In fact, if it is accepted that the elastic modulus of monoclinic zirconia is similar to tetragonal zirconia or few tens of GPa higher [26,27], the sole phase change cannot explain the observed variation.

The compressive strength of non-degraded 3Y-TZP micropillars ( 7.5 GPa) is similar for the different samples tested and much higher than for bulk samples $(2 \times 2 \times 4 \mathrm{~mm})$ processed in the same $A S$ condition, for which the compressive strength was only $\sim 4 \mathrm{GPa}$. This difference is mainly imputable to the absence of processing defects, as reported in Ref. [28]. Even though the micropillars mechanical response might be affected by ion implantation resulting from FIB milling, which creates a superficial amorphous layer of less than $20 \mathrm{~nm}$ in thickness [29], this effect is considered of minor importance for ceramic polycrystals, where dislocation movement is not the main deformation mechanism.

The slope of the stress-strain curve of the AS 3.3 micropillars is initially comparable to that expected for elastic behavior with the bulk elastic modulus of polycrystalline zirconia, whereas a progressive curvature appears as the load increases above $\sim 3 \mathrm{GPa}$. This threshold value depends on the diameter of the micropillar and is generally reduced (lower observed value at $\sim 1.7 \mathrm{GPa}$ ) for sub-micrometric micropillars with diameter of similar dimensions as the grain size [28]. The failure strain calculated from the stress-strain curves for $A S$ micropillars is of about $6.5 \%$, which is considerably high for brittle ceramics, and is related to transformation-induced plasticity. The deviation from linearity between stress and strain at $\sim 3$ GPa in AS 3.3 micropillars may correspond to the stress necessary for inducing stress-activated $t-m$ transformation in favorably orientated individual grains with the presence of the constraints imposed by the neighboring crystals. In sub-micrometric $A S$ micropillars, the reduction of constraints allows the transformation to occur at lower stresses, resulting in the observed instantaneous strain bursts for the $A S 0.3$ micropillars.

The micropillars compressive strength is markedly reduced after degradation to values between 2 and 4 $\mathrm{GPa}$, which are generally lower than for AS macroscopic samples ( 4 GPa). The mechanical response of the degraded micropillars is affected by the presence of degradation microcracks, which are densely present before testing, as shown in Fig. 5. Similarly, no $t-m$ transformation is possible under loading since practically all grains have already been transformed by hydrothermal degradation before testing, as indicated by the XRD results. Even if some residual untransformed volumes might exist inside the degraded 
micropillar, these may not transform since failure is likely to occur before reaching the critical stress for transformation, which is above $3 \mathrm{GPa}$ for $A S 3.3$ micropillars.

Since the cylindrical micropillars have been milled with their axis perpendicular to the degraded surface and degradation microcracks are roughly parallel to the original surface exposed to aging [12,22], a high proportion of them are orientated along planes perpendicular to the compression axis. However, since these cracks are entirely intergranular, they follow the orientation of grain boundaries, so the local crack orientation ranges over different angles with the stress axis.

According to the micromechanical modeling of Nemat-Nasser and Horii [30] and Ashby and Hallam [31], when the load increases in compression, the faces of intergranular microcracks orientated with a suitable angle to the compressive axis tend to slide creating high stresses at the crack tips in mode-II. At these sites, mode-I tension cracks (wing cracks) can nucleate from the existing cracks tip and deviate towards the compression axes. The coalescence of wing microcracks with nearby wing or tilted degradation cracks may be responsible for the observed failure by axial intergranular splitting of the micropillar like on the right side of Fig. 6. The final fracture is completely intergranular with the presence of debris indicating a general low cohesion of the material.

While the above discussion is valid for DEG 3.3 micropillars, for which similar failure stresses and strains are observed among different tests, the behavior of DEG 0.3 micropillars significantly differs from one test to another. Since in the latter case the average grain size $(\sim 350 \mathrm{~nm})$ has similar dimensions to the diameter of the pillar, only few grains and related grain boundaries are present inside the tested volume, so the probability of having microcracks suitably inclined with respect to the compressive axis diminishes. The role of individual grain orientations may also be important for the scatter in the results, although this effect should be similar for AS 0.3 pillars, where the scatter is much lower [27]. Indeed, failure stresses are always lower for DEG 0.3 than for $A S 0.3$, meaning that some microcracks or weakened grain boundaries are always present in the degraded pillars. The statistical occurrence of microcracks or weak interfaces favorably orientated in the volume is therefore responsible for the significant scatter in the results for $D E G$ 0.3. As any of the few possible microcracks present in the volume will always be close to the external surface and their orientation may not favor the formation of wing cracks, the mechanism of fracture may change, shifting from axial splitting to mode-II, as shown in Fig. 7. These aspects could justify the higher strength and strain observed for the sub-micrometric pillars.

Another characteristic of the stress-strain behavior of the DEG 0.3 micropillars is the presence of a series of discontinuous strain bursts until failure, which resemble the strain bursts observed for AS 0.3 micropillars. Some of these events could be also occasionally observed in $D E G 0.7$ samples, although of minor extent in this case. The origin of strain bursts in degraded micropillars may be related to stable crack growth according to the sliding crack model under high compressive stresses, where the effect of friction between crack surfaces may help in avoiding unstable crack propagation. As explained above, the tetragonal- 
monoclinic transformation of untransformed regions is unlikely to occur in this case, while transformation from one monoclinic variant to another has not been observed to our knowledge.

The lower initial stiffness of DEG 3.3 and DEG 0.7 samples against the AS counterparts is also the result of the microcracks network. Under compression, open degradation microcracks and inclined sliding microcracks reduce the stiffness. On the other hand, DEG 0.3 samples have generally higher stiffness, again thanks to the reduced number of microcracks in the volume.

The loading-unloading tests presented in Fig. 3 can be rationalized by noticing that the initial loading slope $S_{0}$ is considerably smaller than the unloading slope at the peak load, $S_{\mathrm{UP}}$, and similar to the slope at the end of full unloading, $\mathrm{S}_{\mathrm{U} 0}$. Stiffness increases during loading due to the increasing contact between opposite faces of open microcracks under compression, which results in a progressively higher effective load-transfer area. At the same time, microcracks that are tilted with respect to the compressive axis tend to first close and then to slide, developing high friction forces between crack faces. At the beginning of unloading, cracks are still closed and the high friction forces hinder the return of the cracks faces to their original position, which justify the higher values of $S_{U P}$. During the progression of unloading, the cracks orientated normally to the compression axis can re-open and friction forces diminish so cracks in mode-II can progressively slide back to their original position, resulting in a reduction in the measured stiffness. Since an increasing fraction of cracks is interested by this mechanism as the peak stress increases, $S_{U P}$ increases accordingly. $S_{0}$ increases slightly too with the peak stress, meaning that the mechanism is not totally reversible, that is, a small fraction of the cracks remain locked. Following the model of Lauterbach et al. [32], the increasing fraction of cracks involved in this process for higher peak stresses is also the reason for the presence of unloading/loading hysteresis loops of increasing width. Because of the higher number of cracks "locked" at high loads for increasing peak stresses, the number of cracks "released" at full unloading is also higher, resulting in decreasing $S_{\mathrm{U} 0}$ values. $\mathrm{S}_{\mathrm{U} 0}$ is in fact an apparent value affected by the occurrence of crack unlocking, while $\mathrm{S}_{0}$ is representative of the stiffness at low stresses. For this reason, their evolution with increasing peak stress is opposite.

Finally, the irreversible component of the strain that occurs at constant peak load (hold time $=10 \mathrm{~s}$ ) shown by the horizontal segments in Fig. 3 may be associated to environmental crack growth as a result of humidity present in the air during testing. This deformation takes place gradually with time and stop after some seconds, in contrast with the instantaneous strain bursts observed in AS 0.3 and DEG 0.3 micropillars. It is well known that the threshold for subcritical crack growth of tetragonal zirconia in the presence of humidity under tension in mode-I is about half the fracture toughness [33]. In degraded pillars, transformation toughening is no longer active, so that the observed effect should be attributed to monoclinic zirconia. However, information on subcritical crack growth in monoclinic zirconia is not available at present. It is interesting to observe that when the peak stress is not maintained, the loading curve deviates from linearity at every stress increase giving a certain irreversible strain contribution (Fig. 3-A). On the contrary, after each hold segment, the following loading curve is rather linear until reaching the next peak 
stress (Fig. 3-B). This evidence suggests an equivalence between time and stress level for subcritical crack growth: the irreversible strain contribution obtained when increasing the stress to a certain value can be

obtained while holding a lower stress during a sufficient time.

\section{Conclusions}

Micro-compression experiments have been performed on FIB-milled micropillars from the zirconia superficial degraded layer after hydrothermal degradation. The compressive failure strength and strain are strongly reduced by degradation, due to the presence of degradation-induced microcracks. Micrometric degraded micropillars present a reproducible mechanical response, where the failure occurs by axial splitting as a consequence of the development and merging of wing cracks from existing microcracks suitably orientated to the compression axis. By reducing the micropillar size in the range of few hundreds of nanometers, the response becomes scattered, which is the consequence of the discrete contribution of a reduced number of weakened grain boundaries and microcracks to the mechanical behavior. Cyclic loading/unloading tests demonstrate that crack closure and sliding under the presence of friction forces in compression are responsible for the observed stiffness changes and the presence of hysteresis loops, while time-dependent deformations may be associated to environmental crack growth.

\section{Acknowledgements}

This work was supported by the Ministry of Economy and Competitiveness (MINECO) under the project ref. MAT2011-23913 and the Government of Catalonia under the project ref. 2014 SGR 130. Erik Camposilvan acknowledges Universitat Politècnica de Catalunya for the grant FPU-UPC, Prof. K. Leifer for his support during the stay at Uppsala University, Prof. U. Wiklund and Dr. J. Heinrichs for their assistance with the nanoindenter, and Dr. T. Trifonov for his help during FIB sessions.

\section{Reference list}

[1] Denry I, Kelly JR. Emerging Ceramic-based Materials for Dentistry. J Dent Res 2014.

[2] Hannink RHJ, Kelly PM, Muddle BC. Transformation Toughening in ZrO2-Containing Ceramics. J Am Ceram Soc 2000;83:461-87.

[3] Miyazaki T, Hotta Y, Kunii J, Kuriyama S, Tamaki Y. A review of dental CAD/CAM: current status and future perspectives from 20 years of experience. Dent Mater J 2009;28:44-56.

[4] Lawson S. Environmental Degradation of Zirconia Ceramics. J Eur Ceram Soc 1995;15:485-502.

[5] Chintapalli RK. Influence of sandblasting on zirconia in restorative dentistry. Universitat Politécnica de Catalunya, 2012.

[6] Gaillard Y, Jiménez-Piqué E, Soldera F, Mücklich F, Anglada M. Quantification of hydrothermal degradation in zirconia by nanoindentation. Acta Mater 2008;56:4206-16.

[7] Hvizdoš P, Chintapalli RK, Valle J, Anglada M. Effect of Low Temperature Degradation on Scratch Behaviour of 3Y-TZP. Key Eng Mater 2009;413:322-5.

[8] Tsubakino H, Kuroda Y, Niibe M. Surface Relief Associated with Isothermal Martensite in Zirconia3-mol\%-Yttria Ceramics Observed by Atomic Force Microscopy. J Am Ceram Soc 1999;82:2921-3. 
[9] Garvie RC, Nicholson PS. Phase analysis in zirconia systems. J Am Ceram Soc 1972;55:303-5.

[10] Muñoz Tabares JA, Anglada M. Quantitative Analysis of Monoclinic Phase in 3Y-TZP by Raman Spectroscopy. J Am Ceram Soc 2010;93:1790-5.

11] Muñoz Tabares JA, Jiménez-Piqué E, Anglada M. Subsurface evaluation of hydrothermal degradation of zirconia. Acta Mater 2011;59:473-84.

12] Jiménez-Piqué E, Ramos A, Muñoz Tabares JA, Hatton A, Soldera F, Mücklich F, et al. Focused ion beam tomography of zirconia degraded under hydrothermal conditions. J Eur Ceram Soc 2012;32:2129-36.

113] Legros M, Gianola DS, Motz C. Quantitative In Situ Mechanical Testing in Electron Microscopes. Mater Res Soc Bull 2010;35:354-60.

14] Greer JR, De Hosson JTM. Plasticity in small-sized metallic systems: Intrinsic versus extrinsic size effect. Prog Mater Sci 2011;56:654-724.

15] Uchic MD, Dimiduk DM, Florando JN, Nix WD. Sample dimensions influence strength and crystal plasticity. Science 2004;305:986-9.

[16] Östlund F, Rzepiejewska-Malyska K, Leifer K, Hale LM, Tang Y, Ballarini R, et al. Brittle-to-Ductile Transition in Uniaxial Compression of Silicon Pillars at Room Temperature. Adv Funct Mater 2009;19:2439-44.

[17] Michler J, Wasmer K, Meier S, Östlund F, Leifer K. Plastic deformation of gallium arsenide micropillars under uniaxial compression at room temperature. Appl Phys Lett 2007;90.

[18] Korte S, Clegg WJ. Discussion of the dependence of the effect of size on the yield stress in hard materials studied by microcompression of MgO. Philos Mag 2011;91:1150-62.

[19] Montagne A, Pathak S, Maeder X, Michler J. Plasticity and fracture of sapphire at room temperature: Load-controlled microcompression of four different orientations. Ceram Int 2014;40:2083-90.

[20] Zhang Y, Han X, Zheng K, Zhang Z, Zhang X, Fu J, et al. Direct Observation of Super-Plasticity of Beta-SiC Nanowires at Low Temperature. Adv Funct Mater 2007;17:3435-40.

[21] Lai A, Du Z, Gan CL, Schuh C a. Shape memory and superelastic ceramics at small scales. Science 2013;341:1505-8.

[22] Camposilvan E, Torrents O, Anglada M. Small-scale mechanical behavior of zirconia. Acta Mater 2014;80:239-49.

[23] Toraya H, Yoshimura M, Somiya S. Calibration Curve for Quantitative Analysis of the MonoclinicTetragonal ZrO2 System by X-Ray Diffraction. J Am Ceram Soc 1984;67:C - 119 - C - 121.

[24] Niihara K. A fracture mechanics analysis of indentation-induced Palmqvist crack in ceramics. J Mater Sci Lett 1983;2:221-3.

[25] Chevalier J, Cales B, Drouin JM. Low-Temperature Aging of Y-TZP Ceramics. J Am Ceram Soc 1999;82:2150-4.

[26] Chan S, Fang Y, Grimsditch M, Li Z, Nevitt M V, Robertson WM, et al. Temperature Dependence of the Elastic Moduli of Monoclinic Zirconia. J Am Ceram Soc 1991;74:1742-4. 
[27] Fogaing EY, Lorgouilloux Y, Huger M, Gault CP. Young's modulus of zirconia at high temperature. J Mater Sci 2006;41:7663-6.

[28] Camposilvan E, Anglada M. Role of size and plasticity in zirconia micropillar compression. Acta Mater 2015.

[29] Kiener D, Motz C, Rester M, Jenko M, Dehm G. FIB damage of Cu and possible consequences for miniaturized mechanical tests. Mater Sci Eng A 2007;459:262-72.

[30] Nemat-Nasser S, Horii H. Compression-induced nonplanar crack extension with application to splitting, exfoliation, and rockburst. J Geophys Res 1982;87:6805.

[31] Ashby MF, Hallam SD. The failure of brittle solids containing small cracks under compressive stress states. Acta Metall 1986;34:497-510.

[32] Lauterbach B, Gross D. Crack growth in brittle solids under compression. Mech Mater 1998;29:8192.

[33] Chevalier J, Olagnon C, Fantozzi G, Cales B. Subcritical crack growth and thresholds in a 3Y-TZP ceramic under static and cyclic loading conditions. Ceram Int 1997;8842:263-6. 
Click here to download high resolution image
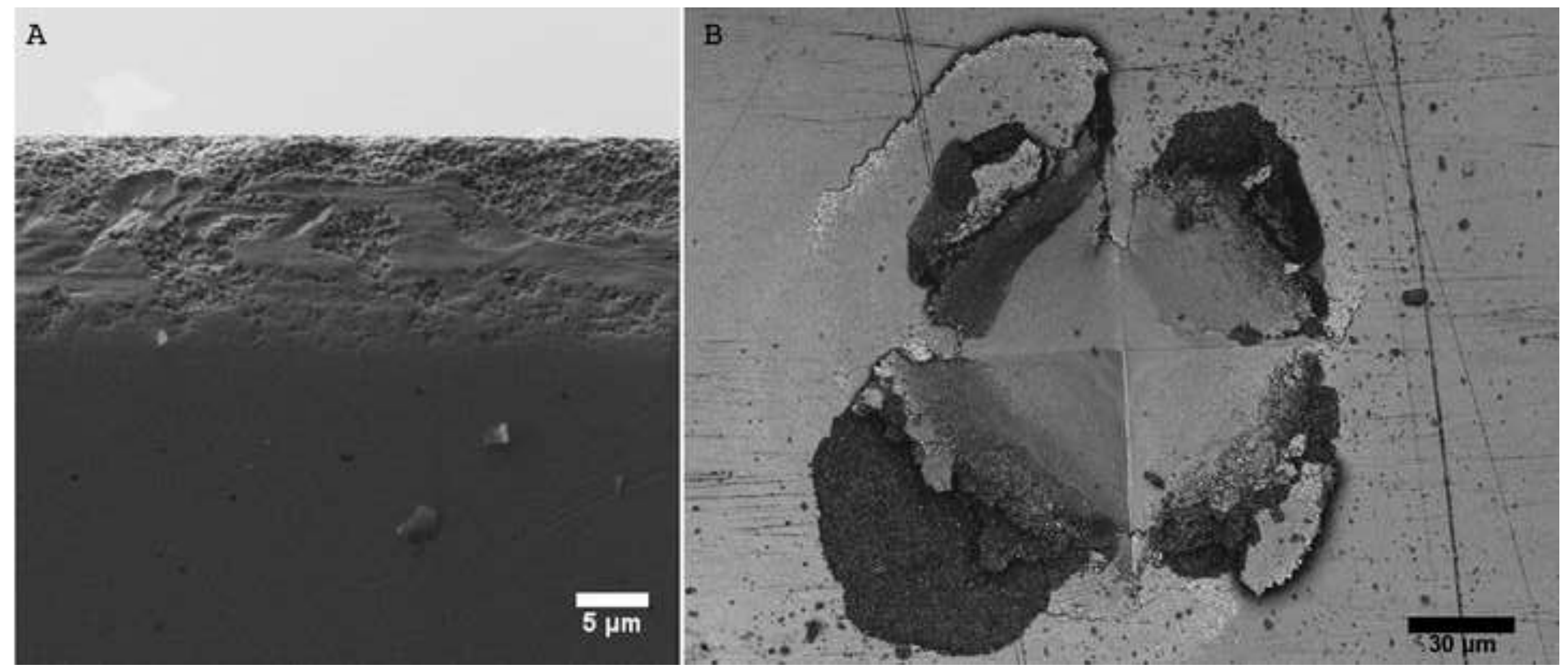

Figure_1 

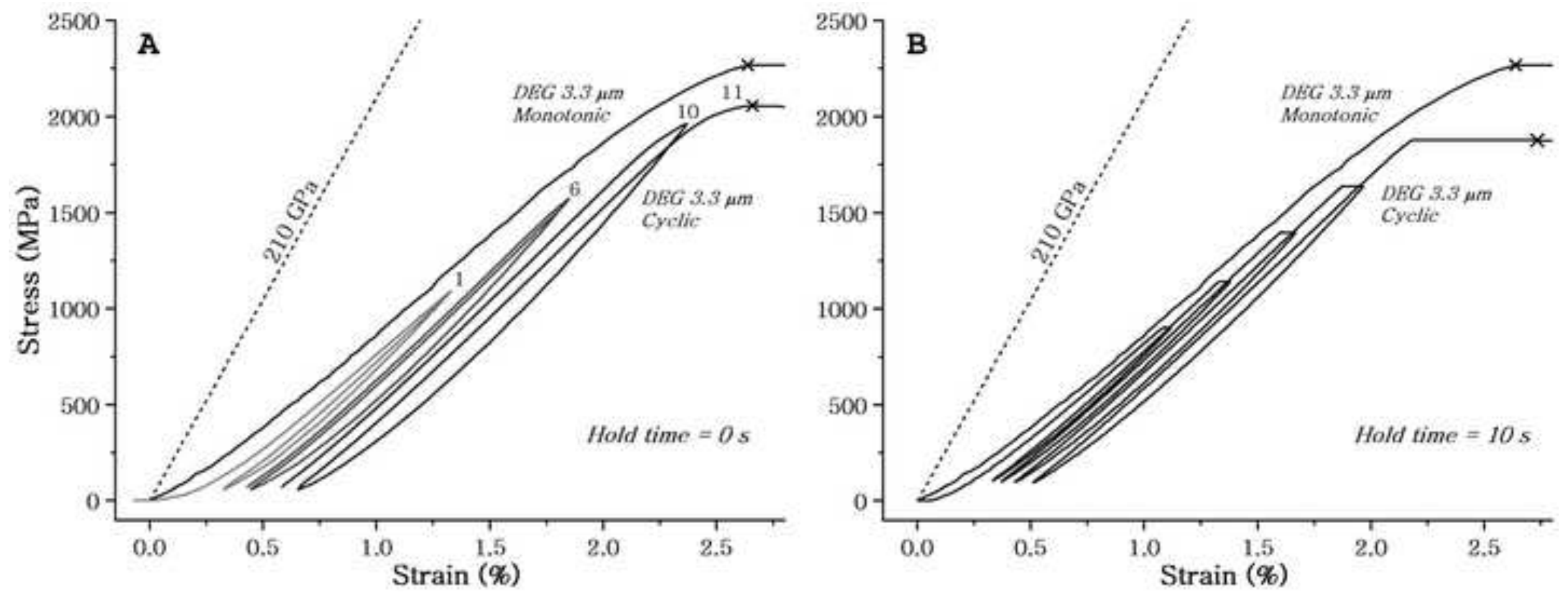
Click here to download high resolution image

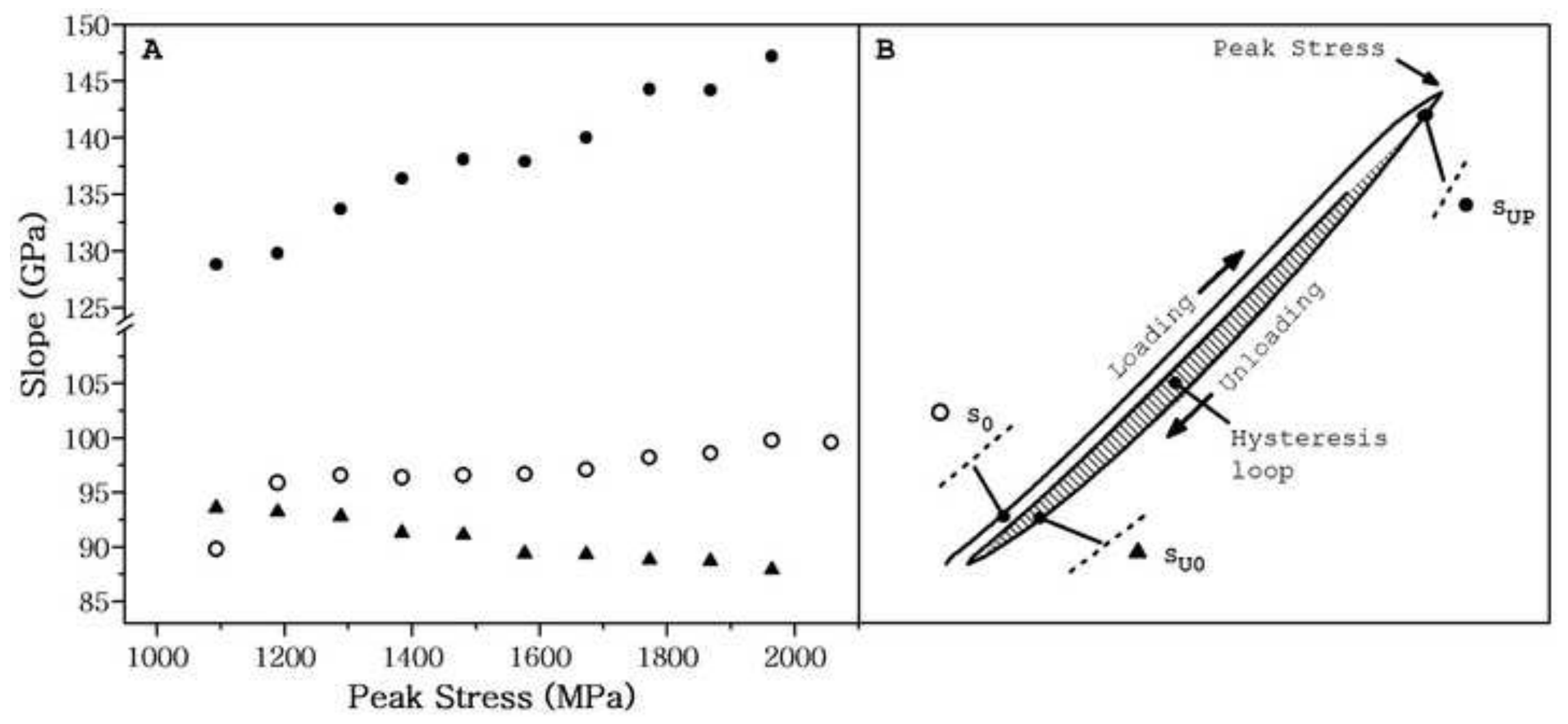


Click here to download high resolution image

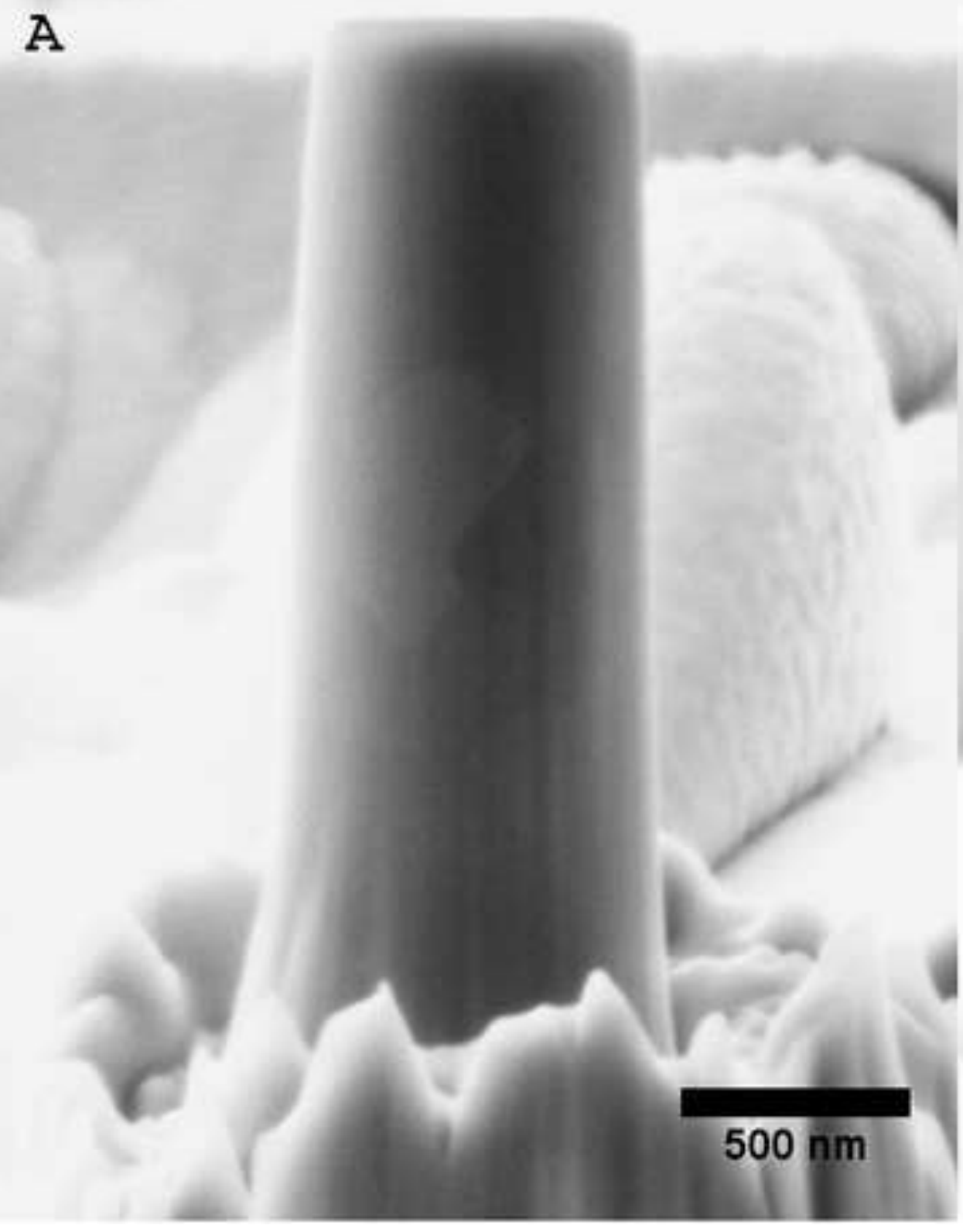

\section{B}


Figure_6

Click here to download high resolution image

4

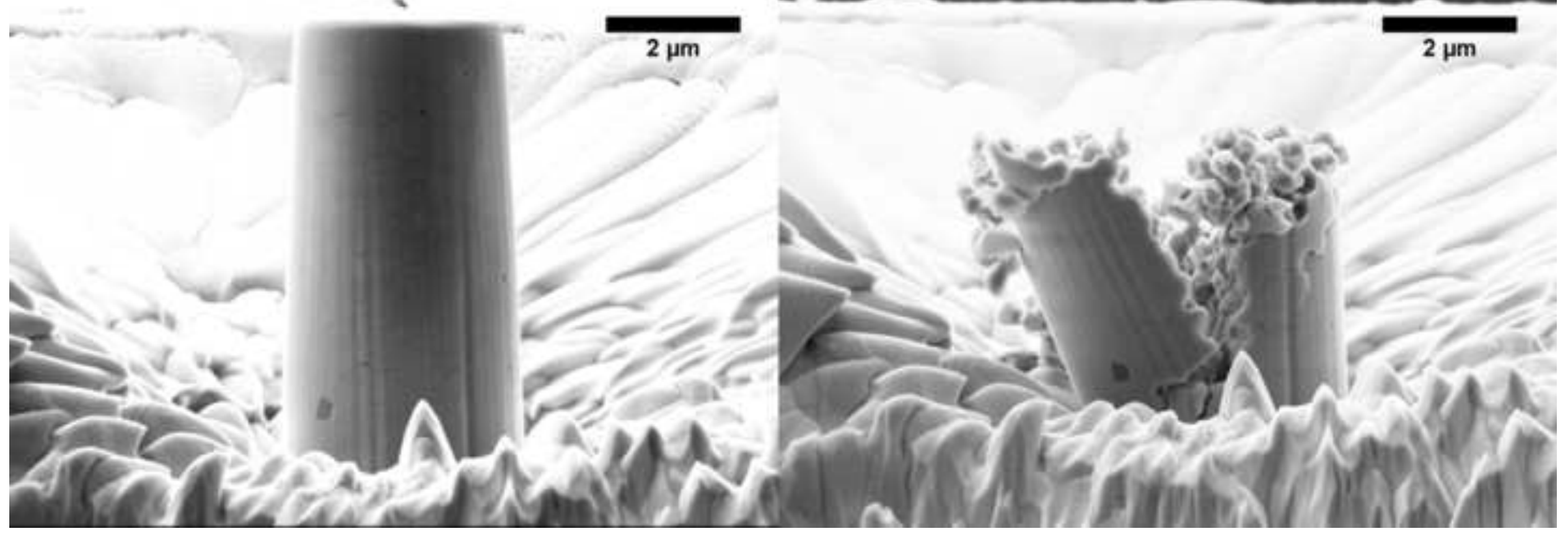


Click here to download high resolution image

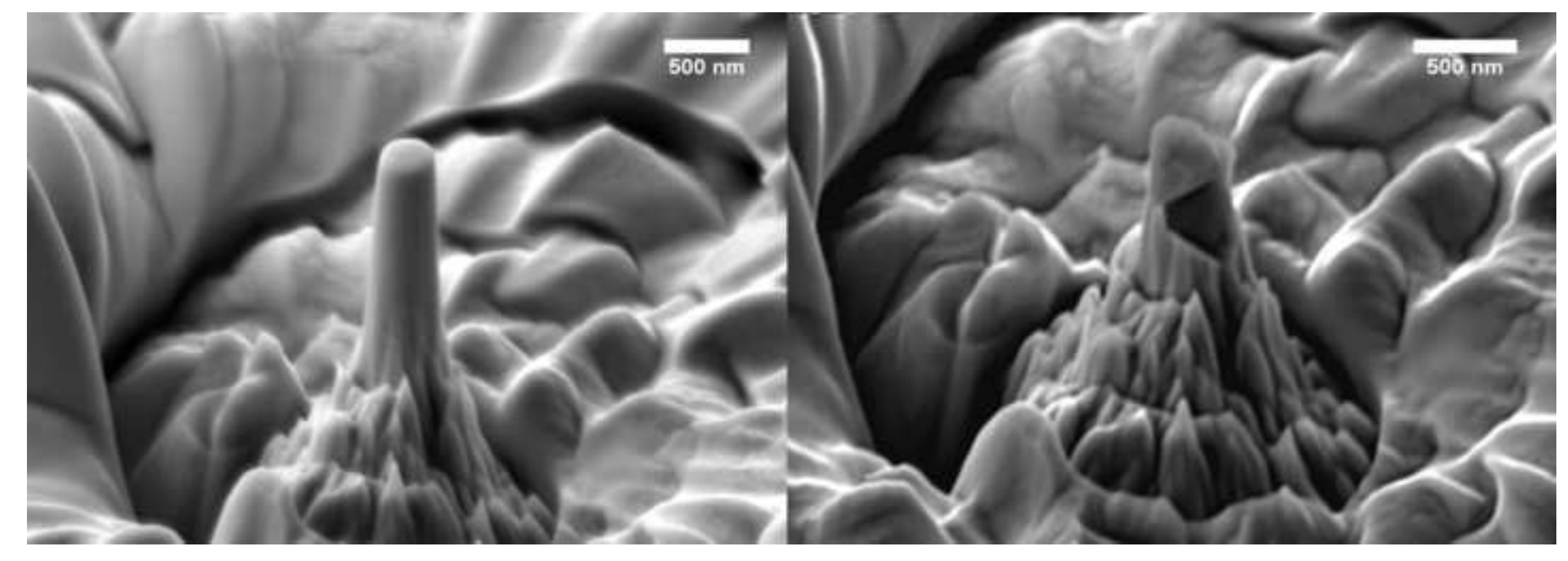


Table 1. Initial properties of as-sintered and degraded zirconia.

Table 2. Size and geometry of tested micropillars.

Figure 1. A: SEM image of the polished cross-section of the artificially degraded disk in the region where the micropillars were milled. The degraded layer presents a granular appearance. Traces of the action of grinding media are also visible inside the degraded layer. B: laser confocal scanning microscopy image of an HV10 indent on the surface of the degraded disk, showing the presence of chipping and debris and the absence of Palmqvist cracks.

Figure 2. Stress-strain curves for degraded and non-degraded micropillars of various diameters. One representative curve is reported for all the samples but the $D E G 0.3$ ones, where 3 curves are presented to show the difference between individual tests. Symbols indicate micropillar failure. The ideal elastic modulus of tetragonal polycrystalline zirconia $(210 \mathrm{GPa})$ is also plotted.

Figure 3. Stress-strain curves for $D E G 3.3$ micropillars by cyclic loading/unloading at increasing peak stress, with (B) and without (A) hold segment at the peak load. Crosses indicate micropillar failure. The ideal elastic modulus of tetragonal polycrystalline zirconia $(210 \mathrm{GPa})$ and a monotonic curve for a micropillar of similar diameter are also plotted.

Figure 4. A: initial loading slope $\left(\mathrm{S}_{0}\right)$, unloading slope at peak load $\left(\mathrm{S}_{\mathrm{UP}}\right)$ and unloading slope at minimum load $\left(\mathrm{S}_{\mathrm{U} 0}\right)$ as a function of the peak load applied in each cycle during cyclic loading at increasing peak stress (Hold time $=0 \mathrm{~s}$ ). The diagram in B shows an example of loading cycle indicating where the slopes were measured.

Figure 5. Lateral surface of one $A S 0.7$ (A) and one $D E G 0.7$ (B) micropillars before testing. Degradation-induced microcracks are clearly observed in the degraded pillar. The vertical striations are due to the milling procedure. Contrast has been enhanced in both pictures.

Figure 6. DEG 3.3 micropillar before and after compression. The intergranular character of fracture can be appreciated on the right picture.

Figure 7. DEG 0.3 micropillar before and after compression. Grain pull-out during testing is evident in the picture to the right. 